\title{
Demanda y provisión de trasporte público en Ciudad Obregón, Sonora
}

\author{
Liz lleana Rodríguez Gámez
}

Resumen: desde hace un par de años, los sistemas de trasporte público enfrentan una crisis que se ha traducido en una disminución de la calidad del servicio y en la degradación o reducción de la flota, por ello algunas ciudades afrontan problemas serios de demanda que exceden la capacidad de dichos sistemas. Uno de los principales obstáculos para hacer frente a esta problemática es la falta de información sobre los patrones de movilidad de los residentes. Por ello, el objetivo de este trabajo es cuantificar el volumen de viajes y derivar la demanda potencial de trasporte público en Ciudad Obregón, Sonora, a fin de proporcionar datos básicos para su planificación y provisión. La metodología emplea un sistema de información geográfica, con información secundaria para determinar las necesidades de movilidad, a partir de la estructura familiar, sus actividades cotidianas y la accesibilidad a los modos de trasportación públicos y privados.

Palabras clave: modos de trasporte; trasporte público; oferta de trasporte público; demanda de trasporte público; provisión de trasporte público; accesibilidad al trasporte público.

* Profesora investigadora del Centro de Estudios del Desarrollo de El Colegio de Sonora (COLSOn). Av. Obregón \#54, colonia Centro, C. P. 83000, Hermosillo, Sonora, México. Teléfono: (662) 259 5300. Correo electrónico: lrodriguez@colson.edu.mx 
Abstract: since a couple of years ago public transportation systems are facing a crisis, as a result of a fall in the quality of the service, as well as a degradation or reduction of the fleet. As a consequence, some cities face serious problems because demand exceeds the capacity of such systems. One of the main obstacles to address this problem is the lack of information on mobility patterns of residents. Thus, the aim is to quantify the volume of travel and derive the demand for public transportation in Ciudad Obregon, Sonora, to provide basic data for its planning and provision. The methodology employs a Geographic Information System (GIS), with secondary information in order to determine mobility needs based on family, its daily activities and accessibility to modes of public and private transportation.

Key words: modes of transportation; public transportation; public transportation supply; public transportation demand; public transportation provision; accessibility to public transportation.

\section{Introducción}

En América Latina, el trasporte público es el modo de desplazamiento más importante para los grupos de bajos ingresos. En Bogotá, Caracas, Lima, Montevideo y la Ciudad de México, éste provee más de 50 por ciento del total de los viajes, y el uso del autobús a diésel es el tipo de vehículo que predomina en el sistema público urbano (Corporación Andina de Fomento, CAF 2011). Pese al aumento de la tasa de motorización privada, que alcanza un crecimiento entre 15 y 20 por ciento anual (Secretaría de Tránsito y Transporte de Bogotá 2012, 8-12), el trasporte público en muchos países enfrenta una demanda creciente pero, al mismo tiempo, se registra un deterioro del sistema debido a que en periodos largos la inversión es poca o nula (Illes 2005).

De hecho, los sistemas de trasporte público urbano en las ciudades latinoamericanas están en crisis permanente debido a problemas de informalidad, bajos estándares de calidad y degradación física de la 
flota vehicular, entre otros (Figueroa 2005; Illes 2005). Al parecer, un mal desempeño económico y diversos problemas de planeación han generado una demanda que excede la capacidad de la mayoría de ellos. Esta situación deriva en una provisión frágil, que pone al sistema en crisis cada vez que algún acontecimiento modifica ligeramente sus condiciones medias de operación.

El servicio de trasporte urbano es necesario para que la población se desplace de un lugar a otro, y satisfaga sus necesidades de movilidad, que varían mucho entre lugares y grupos sociales. De acuerdo con el Observatorio de Movilidad Urbana de América Latina, las ciudades de los países desarrollados tienen más movilidad personal (viaje/persona/día) porque tienen mayores ingresos y los trabajadores participan más en el mercado laboral formal. Por ejemplo, Chicago y Londres registran 4.0 y 2.8 viajes diarios por habitante, en comparación con 2.5 de la Ciudad de México y 2.0 de Buenos Aires (CAF 2011, 2).

Las diferencias en el índice de movilidad de estas grandes urbes están relacionadas con un patrón de actividades sociales y económicas distinto al de otras más pequeñas del sistema urbano mexicano. Por ejemplo, entre las ciudades latinoamericanas de tamaño medio (de hasta 500 mil habitantes), dicho índice suele situarse en 1.6 viajes/ persona/día y en 1.8 en las de hasta 1 millón de habitantes (Vasconcellos 2010, 151). En el contexto mexicano, una encuesta sobre empleo y calidad de vida permitió estimar un índice de movilidad de 1.8 viajes/persona/día (COLSON 2009), para los residentes de Ciudad Obregón, Sonora, que tiene 300 mil habitantes.

En ciudades en vías de desarrollo, el crecimiento rápido de la población es el principal problema para el trasporte público (Illes 2005); sin embargo, en las de América Latina la provisión del servicio debe adaptarse no sólo a las variaciones en la demanda, sino a las nuevas condiciones que enfrenta el trasporte urbano, el cual debe: a) asimilar e integrar los cambios para implementar sus políticas; b) enfrentar una aguda competencia por el aumento en el uso de automóvil privado y otras formas precarias e inseguras de trasporte colectivo y c) habituarse a nuevas estructuras de viajes y a la cobertura de áreas urbanas más extensas, pero con menor densidad de demanda (Figueroa 2005). 
Sin duda, estas condiciones están presentes en mayor o menor grado en las ciudades mexicanas, y actúan como potenciadores de las crisis. En Ciudad Obregón esto se ha manifestado en inestabilidad de la oferta del servicio de trasporte público, en detrimento de la calidad del servicio y en pérdida de aforo (Minjares 2012a y 2012b; Valdivia 2012).

En contrapartida, se registró un aumento del parque vehicular privado de 6.1 por ciento anual en el periodo 1999-2012, mientras que la población lo hizo en 1.7, del año 2000 a 2010 (Rodríguez-Gámez y Tong 2012, 178). Este último aspecto resulta contrastante, pues el número de autobuses en servicio ha estado disminuyendo (Minjares 2012c), lo que, de acuerdo con Landeros (2014), se puede atribuir a la forma de regulación derivada de decisiones políticas inapropiadas y a criterios administrativos de las empresas que proveen el servicio. En este aspecto se estima, como regla general, que para las ciudades en países en vías de desarrollo haya un autobús convencional por cada dos mil habitantes, siempre y cuando éste sea el único modo disponible para la trasportación en el sistema público (Illes 2005). Por ello, se podría estimar que para Ciudad Obregón, con una población aproximada de 300 mil habitantes (Instituto Nacional de Estadística y Geografía, INEGI 2010a), el número óptimo de autobuses sería de 150, cantidad similar a la que prevalecía en 2012, con una flota de 154 (Valdivia 2012; Minjares 2012b).

No obstante, la demanda final del servicio depende de las necesidades de movilidad de la población y de los patrones de comportamiento, los cuales varían sustancialmente según el tamaño y la estructura de la ciudad, los ingresos y la tasa de motorización, la congestión del tránsito y las velocidades promedio, entre los factores más importantes. ${ }^{1}$ Por ello, la estimación y la calibración de la flota óptima debe realizarse tomando en cuenta estas consideraciones; por ejemplo, en las zonas metropolitanas de León (1.6 millones de habitantes) y Guadalajara (4.4) el número de habitantes por autobús fue de 760 y 933 pasajeros (CAF 2009, 20-21).

1 Consultar Rodríguez-Gámez y Tong (2012), para una referencia de los aspectos que incluyen las decisiones de los viajeros en el caso de Ciudad Obregón. 
Estas cifras dan muestra de los parámetros de cobertura, pero no de la calidad del servicio, y generan debate en torno a la flota que debe prevalecer en Ciudad Obregón, para prestar el servicio de acuerdo con las necesidades de movilidad de la población. Por ejemplo, para las autoridades estatales el número ideal debería ser de 230 autobuses (Secretaría de Gobierno del Estado de Sonora 2012). En cambio, las empresas que tienen concesionado el servicio en Ciudad Obregón estiman 200 unidades para ofertar adecuadamente el servicio. ${ }^{2}$ Estas inconsistencias reflejan la falta de información y planeación del sistema de trasporte, y constituye uno de los temas críticos que requiere directrices de investigación, pues la demanda es desconocida incluso para las empresas que proveen el servicio, que no cuentan con un método de aforo, ni encuestas origen-destino para estimar la demanda real.

Así, la planeación del sistema de trasporte público (oferta y demanda), con información veraz, contribuirá en la formulación de políticas públicas que respondan a las necesidades de los usuarios. Es por ello que los objetivos de este trabajo son: a) estimar, según las necesidades de movilidad, el número de viajes que se origina en cada área de la ciudad; b) analizar la cobertura del servicio y, en consecuencia, c) derivar la demanda potencial y la oferta en la provisión del servicio de trasporte público. La metodología utilizada parte de la creación de un sistema de información geográfica (SIG), así como de la identificación de las necesidades de movilidad de la población, para lo que se consideran las características de los viajeros, si cuentan con automóvil y las condiciones laborales, para estimar el volumen de viajes/viajeros y usuarios del trasporte público en la ciudad.

En la segunda sección de este artículo se ofrece una revisión de los elementos teóricos, que permiten relacionar las características urbanas y tendencias en la movilidad con la demanda y oferta de trasporte urbano. En la tercera se describe el área de estudio, que se centra en las características urbanas, en las del sistema de trasporte público y en la movilidad de la población. En la cuarta se detallan los datos y el enfoque metodológico adoptado para la estimación de la demanda de

2 Comparecencia de los representantes legales de las empresas de trasporte de Ciudad Obregón en sesión ordinaria del Consejo Ciudadano del Transporte Sustentable del Estado de Sonora. El Colegio de Sonora, Hermosillo, Sonora, agosto de 2014. 
trasporte urbano. La quinta contiene los resultados de la estimación de la demanda y su cobertura en las áreas urbanas, y se concluye con algunos comentarios en torno al sistema de trasporte público en Ciudad Obregón.

\section{Marco teórico}

Movilidad y accesibilidad

La movilidad se refiere a la capacidad o cantidad de movimientos que las personas realizan en un sistema específico o en un área socioeconómica, que incluyen el espacio y el tiempo, los motivos y el modo de trasporte utilizado (Jaramillo et al. 2012). La movilidad urbana se asocia con los desplazamientos recurrentes de la población en la ciudad, para acceder a bienes y servicios (Miralles-Guasch y Cebollada 2003). En este sentido, la necesidad de movimiento de las personas origina el trasporte a lo largo de un espacio geográfico, para la realización de actividades como ir al trabajo, a la escuela, de paseo o de compras. Así, los modos de trasporte como caminar, tomar un autobús o conducir un automóvil son mecanismos que posibilitan la movilidad urbana.

La economía urbana ha estudiado la movilidad a partir de dos perspectivas: a) en su relación con la estructura urbana y las consecuencias (externalidades) de desplazamientos tardados, largos y b) el análisis y modelación de las decisiones o conducta de los viajeros o commuters (Rouwendal y Nijkamp 2004; Schwanen et al. 2004). En ambos enfoques, el estudio de los modos de trasporte aparece como un elemento interviniente mucho más acotado que el de movilidad. Este último es un concepto más relacionado con el de calidad de vida, por lo que desde el punto de vista social los estudios deben resaltar el acceso equitativo, no excluyente, a los modos e infraestructuras de trasporte (Flechas 2006; Lazo 2008; Casado 2014; Negrete y Paquette 2011).

Como tal, la movilidad urbana "ofrece una perspectiva de los individuos en su realidad socioeconómica y espacial (edad, género, categoría socio-laboral) más amplio que el término transporte, el cual se limita a una relación de oferta y demanda expresada esquemática- 
mente, por un lado, en cantidad de infraestructuras y, por el otro, en el número de desplazamientos por persona por día, según motivo, modo, itinerario, tiempo" (Montezuma 2003, 175). En este contexto debe analizarse el acceso diferenciado y desigual que los grupos de población tienen en relación con los modos de trasporte (Casado 2014).

Por ello: "La accesibilidad hace referencia a la facilidad con que cada persona puede superar la distancia que separa dos lugares y de esta forma ejercer su derecho como ciudadano. La relativa facilidad para superar la distancia es una variable relacionada con las características físicas de un espacio, las oportunidades de uso de ciertas actividades y las características individuales de los ciudadanos" (Miralles-Guasch y Cebollada 2003, 14). De ahí que la accesibilidad no sea uniforme para todos los habitantes en un mismo espacio. Por lo que no puede hablarse de una accesibilidad genérica. Esta perspectiva ha tenido un cierto desarrollo en América Latina, donde el carácter multidimensional de la exclusión social se manifiesta en el acceso diferenciado y desigual a medios y mecanismos de movilidad urbana, como en el caso del trasporte urbano de carácter público.

Demanda de trasporte público

La demanda de trasporte se deriva de la cantidad de población que viaja con el fin de satisfacer una necesidad (por ejemplo el trabajo, la escuela, el ocio, la salud) o para realizar una actividad en algún lugar. Por ello, hay toda una gama de demandas específicas para el trasporte que son altamente cuantitativas y diferenciadas, según la hora, el día de la semana, el propósito del viaje, el tipo de carga, la importancia de la velocidad y la frecuencia (Ortúzar y Willumsen 2011). Esta característica hace que la demanda de trasporte sea más difícil de analizar y pronosticar, porque involucra un espacio y una distribución de las actividades en él, que es la razón de su existencia (Ortúzar y Willumsen 2011); por lo tanto, el tamaño y la estructura urbana de la ciudad tienen un efecto en la demanda por trasporte público. Un aumento en el policentrismo (la existencia de varios centros de empleo) se asocia con un incremento en el uso del automóvil y, por tanto, con una reducción de la importancia de los modos colectivos/públicos de 
trasporte (Buchanan et al. 2006; Schwanen et al. 2004), de ahí que en ciudades monocéntricas (donde hay un solo centro de empleo) la demanda tiende a ser mayor. Así el uso del auto se asocia con viajes más cortos, mientras que los del trasporte urbano suelen ser más largos (Gordon y Richardson 1996; Vega y Reynolds-Feighan 2008; Cebollada 2009).

Además, la oferta y la demanda de trasporte tienen elementos dinámicos muy fuertes (Ortúzar y Willumsen 2011). Una buena parte de la demanda se concentra en pocas horas del día (las pico), de tal manera que se observan variaciones por hora y día de la semana, especialmente en los viajes al trabajo y a la escuela en horarios muy específicos y durante ciertos días de la semana (Balcombe et al. 2004). Por ello, podría ser que un sistema de trasporte funcione bien considerando la demanda promedio de viajes en un área, pero que entre en crisis en los periodos de hora pico (Ortúzar y Willumsen 2011).

Para quienes viven en la periferia de la ciudad, los recorridos suelen ser más largos, ya que el trasporte colectivo/público es menos eficiente, por la baja densidad de población en estas áreas (Vega y Reynolds-Feighan 2008). Por esto el uso del trasporte público está asociado con la falta de acceso al automóvil (Balcombe et al. 2004). Además, los ingresos influyen mucho en el uso de diversos modos de trasporte; por ejemplo, los salarios de los peatones, ciclistas y usuarios de trasporte público suelen ser bajas (Vasconcellos 2010).

Tanto hombres como mujeres de un rango amplio de edades, con ingresos distintos y también con tasas diferentes de propiedad de autos, y que viven en diversas áreas de la ciudad utilizan el servicio de trasporte público, para una gran variedad de propósitos. Por ello, se debe considerar cómo incide el ingreso, la edad, el género y la estructura familiar de los usuarios en las variaciones de la demanda. Al respecto, Vasconcellos (2010), apoyado en evidencia para ciudades de países en vías de desarrollo, menciona las siguientes regularidades en las necesidades de las personas, que se deben tener en cuenta en la planificación del trasporte:

- La edad está relacionada primordialmente con el trabajo, por ello quienes están en edad productiva se desplazan más. Los niños y jóvenes también se consideran muy móviles por la importancia 
de sus traslados a la escuela. Mientras que los niños pequeños, quienes suelen ir acompañados por los mayores, y los jubilados se mueven menos.

- En la mayoría de las sociedades, debido a la división de tareas del hogar, las mujeres son menos móviles que los hombres; además, ellas suelen caminar más y usan menos el trasporte motorizado.

- Los hombres suelen viajar más que las mujeres, también los adultos involucrados en actividades laborales se desplazan más que los jóvenes y los de edad avanzada.

- Las personas con alguna discapacidad física son menos móviles, por razones obvias, en países en desarrollo es raro observar algún tipo de apoyo por parte del gobierno, por lo que es el grupo social más perjudicado en sus necesidades de movilidad.

La oferta de trasporte

La característica distintiva de un sistema de trasporte es que ofrece un servicio y no constituye un bien, por lo tanto no es posible almacenarlo y se debe consumir en el momento y lugar en que se produce, de lo contrario se pierde el beneficio (Ortúzar y Willumsen 2011). Además, requiere activos fijos, como la infraestructura y unidades móviles, los vehículos. La combinación de estos elementos, junto con un conjunto de reglas de operación, es lo que posibilita el desplazamiento de los usuarios.

Así, el servicio debe estar representado por condiciones promedio, que se ofrezcan en términos de la ocupación (comodidad), de las posibilidades efectivas de embarque (tiempo de espera, que incluye la macro accesibilidad) y de la calidad de la señalización e información disponibles para los usuarios (Vasconcellos 2010, 63). Además, existe una amplia gama de modos de trasporte con variaciones considerables en la tecnología, el costo, las características de funcionamiento, la extensión geográfica, la cuota de mercado y la composición socioeconómica de los viajeros (Pucher 2004).

Entre los medios urbanos más utilizados están los privados motorizados (auto), los públicos motorizados (autobús, subterráneos o sistemas de autobús de tránsito rápido) y los no motorizados (caminar, la bicicleta). No obstante, en la actualidad se registra un dominio 
de los desplazamientos en modos privados motorizados, en detrimento del público motorizado y los no motorizados (Balcombe et al. 2004). Sin embargo, en ciudades de países en vías de desarrollo, la importancia del trasporte público continúa siendo considerable debido a los bajos ingresos de la población.

Por lo general, los modos colectivos de trasporte incluyen los servicios de ruta fija para todos los usuarios, de ahí su carácter de trasportación pública. Puede ser suministrado tanto por empresas públicas como por privadas, y comprende los medios en que los pasajeros no son los propietarios, sino que se trata de un servicio proporcionado por terceros. Aun cuando la forma más común es el autobús, existe el "para-tránsito", conformado por flotas de camionetas vans, taxis y otros sistemas "puerta a puerta", que son demandados (Pucher 2004); también está el autobús escolar, que proporciona un servicio importante. ${ }^{3}$

La flota de trasporte urbano, por ejemplo, está determinada por el tamaño de la ciudad, por los patrones en la movilidad de sus habitantes y por la disponibilidad de otros medios. Al respecto, Vasconcellos $(2010,151)$ estimó el consumo de viajes y provisión del servicio para ciudades típicas, en función del tamaño de la población; así, para las de menos de 80 mil habitantes la flota recomendada es de 60 unidades (1 335 habitantes por autobús), mientras que para una media, de 500 mil, la flota ascendería a 400, y a 800 unidades (1 250 habitantes por autobús) en las grandes, de un millón de habitantes.

Un elemento que distorsiona la provisión del servicio de trasporte es el de los efectos secundarios como los accidentes, la contaminación y la degradación del medio ambiente, entre otros, que el usuario rara vez percibe o paga los costos de limpieza del medio ambiente o el cuidado de los heridos en accidentes relacionados con él (Ortúzar y Willumsen 2011). Otra característica importante de la oferta es la congestión que surge cuando la demanda se acerca a la capacidad instalada, y el tiempo requerido para utilizarla supera por mucho la media en condiciones de baja demanda.

3 El para-tránsito y los autobuses escolares atienden por naturaleza a grupos especiales con un propósito específico de viaje, y ofrecen rutas y horarios flexibles dependiendo de la demanda de los pasajeros. 


\section{Patrones de movilidad en Ciudad Obregón}

Crecimiento del parque vehicular privado

En las últimas décadas se han registrado cambios importantes en los patrones de movilidad en las zonas urbanas, lo cual se ha traducido en un uso creciente del vehículo privado. Entre las principales ciudades de Sonora, la tasa de motorización de vehículos privados (número de habitantes por automóvil) es mayor en Ciudad Obregón que en Hermosillo (pese a que esta última es más grande y es la capital del estado), incluso supera la de Nogales (véase figura 1). La tasa de motorización en Ciudad Obregón aumentó de 1980 a 2010, pasó de 22 a 5 personas por auto (véase figura 1), registrando el proceso de motorización más rápido. ${ }^{4}$ En términos comparativos, la información de Hermosillo y Ciudad Obregón sugiere una relación positiva entre el tamaño de la ciudad y la tasa de motorización.

\section{Figura 1}

Tasa de motorización de vehículos privados (habitantes por automóvil)

\begin{tabular}{|c|c|c|c|c|c|}
\hline Año & México & Sonora & Hermosillo* $^{*}$ & Ciudad Obregón & Nogales \\
\hline 1980 & 16.92 & 17.48 & 13.80 & 22.08 & 6.13 \\
\hline 1990 & 12.39 & 11.63 & 11.23 & 15.22 & 5.46 \\
\hline 2000 & 9.58 & 7.68 & 7.25 & 8.33 & 3.65 \\
\hline 2010 & 5.31 & 4.56 & 4.02 & 5.30 & 2.77 \\
\hline
\end{tabular}

* Considera información municipal.

** La información se refiere al total para el municipio de Cajeme, del cual Ciudad Obregón es la cabecera.

Fuente: elaboración propia, con base en los censos de población y registros administrativos del INEGI (2014).

4 Este uso creciente del vehículo privado, del que dan cuenta las estadísticas, se puede considerar excesivo si se suman los datos de los autos que han ingresado al país y que aún no están regularizados o registrados. Por ejemplo, en Nogales, como ocurre en otras ciudades fronterizas, 86 por ciento de los automóviles son estadounidenses y 14 mexicanos (INE 2009, citado en Rosales 2012, 80), lo cual supera la tasa de motorización de las áreas metropolitanas de México. 
La tasa de motorización, que puede calificarse como un indicador global, no da cuenta plena de las necesidades de movilidad de la población; por ello se considera ahora la tasa de propiedad de autos (número por vivienda). Así, se observó que en las principales ciudades de Sonora aumentaron los autos por vivienda, llama la atención el caso de Nogales (véase figura 2). Cabe resaltar que en Ciudad Obregón hay menos personas que tienen auto, situación que requiere atención rápida, pues existe una demanda mayor por modos colectivos de trasporte como el autobús urbano; mientras que en Hermosillo y en Nogales las estadísticas muestran que existe, en promedio, al menos un auto por vivienda habitada (véase figura 2).

\section{Figura 2}

Tasa de propiedad de autos por vivienda habitada

\begin{tabular}{|c|c|c|c|c|c|}
\hline Año & México & Sonora & Hermosillo* $^{*}$ & Ciudad Obregón $^{* *}$ & Nogales $^{*}$ \\
\hline 1980 & 0.33 & 0.31 & 0.40 & 0.26 & 0.82 \\
\hline 1990 & 0.41 & 0.41 & 0.42 & 0.32 & 0.88 \\
\hline 2000 & 0.47 & 0.55 & 0.57 & 0.50 & 1.17 \\
\hline 2010 & 0.75 & 0.83 & 0.93 & 0.69 & 1.41 \\
\hline
\end{tabular}

* Considera información municipal.

** La información se refiere al total para el municipio de Cajeme, del cual Ciudad Obregón es la cabecera.

Fuente: elaboración propia, con base en los censos de población y registros administrativos del INEGI (2014).

La trasportación urbana

En la figura 3 se muestra la importancia relativa de los principales modos de trasporte público en Ciudad Obregón, donde el autobús urbano proveyó 37.1 por ciento de todos los servicios en 2008, seguido por el auto (25.3), los traslados a pie (16.2) y como pasajero en auto privado (10.8). Se revisó la elección del modo de trasporte por categoría ocupacional, ya que para los trabajadores es un tema crítico, pues contar con un automóvil aumenta la posibilidad de conseguir 


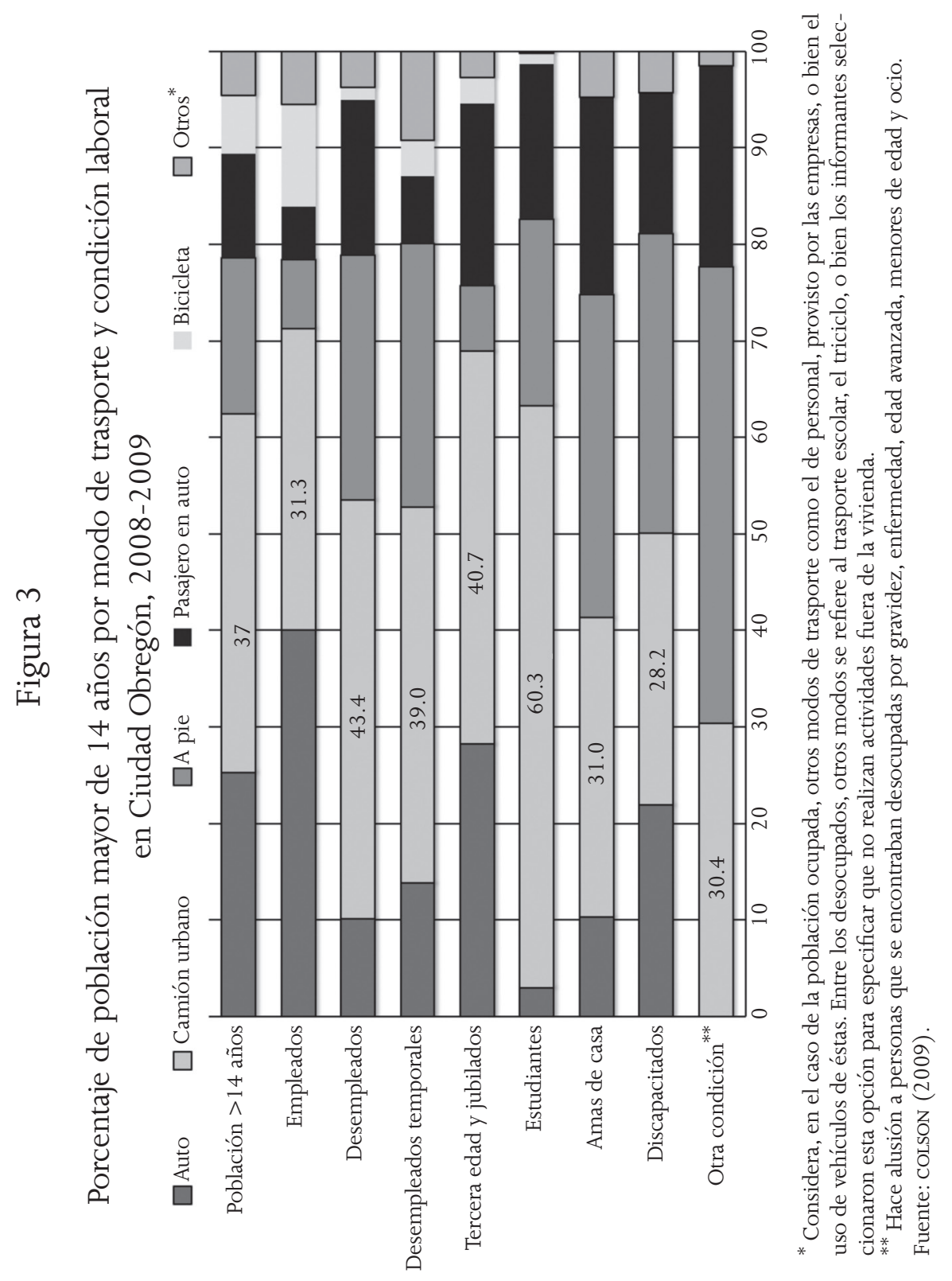


un trabajo o disminuir las tasas de desempleo (Gautier y Zenou 2010; Baum 2009).

Cuando se tienen en cuenta las condiciones laborales de la población algunas diferencias son evidentes. Por ejemplo, los empleados utilizan principalmente el trasporte privado motorizado (50.1 por ciento), que comprende los viajes en auto (40.0), como pasajero en un auto (5.5) y otros motorizados (4.6); mientras que los desempleados sólo usan los modos privados motorizados, en 29.9 por ciento de sus viajes. Por otro lado, 31.3 por ciento de los empleados se trasladan en autobús urbano, contra 43.4 de los desempleados, para quienes es el modo de trasporte principal (véase figura 3).

Para los estudiantes, el trasporte público es especialmente importante, 60.3 por ciento de ellos lo utilizan para desplazarse del hogar a la escuela, seguido por los traslados a pie en 19.3 de los casos. En orden de importancia, a este grupo le sigue el de la tercera edad y los jubilados, quienes usan el autobús urbano en 40.7 por ciento de las ocasiones. Al menos una tercera parte de amas de casa y los desocupados por otra condición (gravidez, enfermedad, edad avanzada, menores de edad y ocio) también son usuarios del trasporte público (véase figura 3 ).

Es preciso señalar que el auto ofrece a los trabajadores, tanto ocupados como desocupados, ciertos beneficios que el trasporte público no tiene. Por ejemplo, la ampliación geográfica para buscar un empleo facilita el traslado a uno que esté más lejos de casa, y a otros que requieran jornadas de trabajo poco usuales. No obstante, la característica más valorada es la reducción del tiempo de viaje, en comparación con el que les tomaría hacerlo en el trasporte público (Baum 2009); en Ciudad Obregón es de ocho minutos más (véase figura 4). ${ }^{5}$ Sin embargo, hay diferencias muy marcadas en función de los horarios; por ejemplo, de 15:00 a 20:00 horas el viaje en auto toma 57 minutos en promedio, mientras que en autobús urbano al mediodía (de 9:00 a 15:00 horas) se demora más que en otro horario, llega a los $52 \mathrm{mi}-$ nutos en promedio por viaje (Rodríguez-Gámez y Tong 2012, 179).

5 Esta información es similar a los hallazgos en ciudades latinoamericanas, con tiempos de 30 minutos en auto y hasta 49 en trasporte público (CAF 2009, 13); mientras que en México el promedio fue de 35 minutos (INEGI 2009). 


\section{Figura 4}

Tiempo promedio de traslado (viaje sencillo) al trabajo (mercado estructurado) por modo de trasporte en Ciudad Obregón, 2008-2009

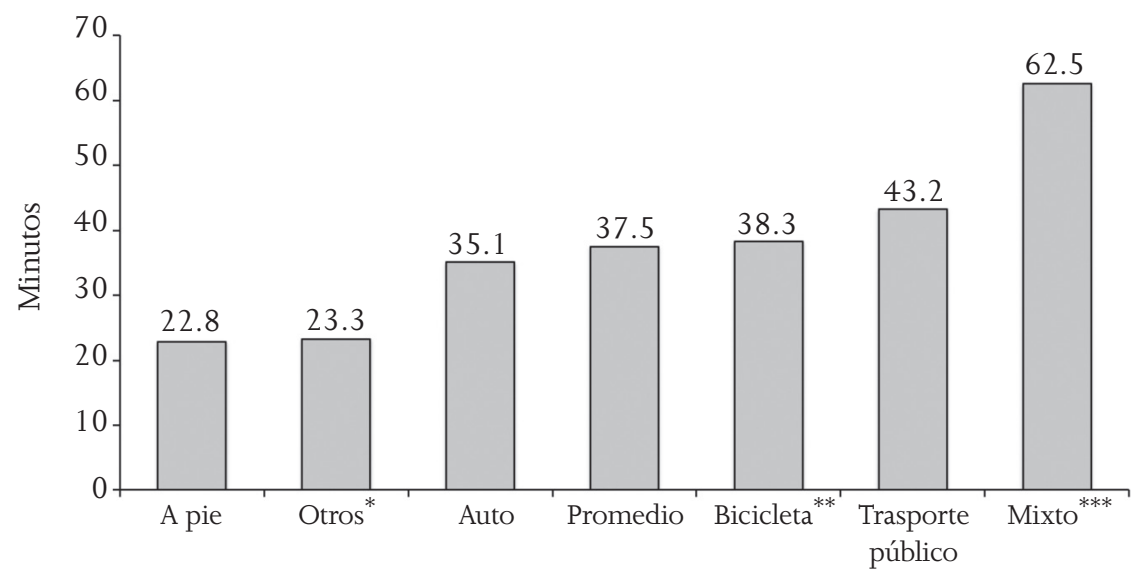

* Traslados en carreta, carruaje, caballo o burro.

** Traslados en trasportes de tracción: bicicleta, triciclo o bote de remos.

*** Viajes en los cuales se combinó más de un modo de trasporte.

Fuente: elaboración propia, con base en COLSON (2009).

El sistema de trasporte público

La estructura monocéntrica de Ciudad Obregón implica que la mayoría de los empleos se localizan en el centro de negocios (central business district, CBD, por sus siglas en inglés); la densidad de empleo decrece conforme se va alejando de este punto. Las personas que viven alrededor del CBD están en relación opuesta a la distancia. Concentrar los puestos de trabajo en un solo centro de empleo provoca presiones de congestión en las arterias viales principales que conducen a él, y se deja sin utilizar la capacidad en las rutas tangenciales y circunferenciales. Además, la centralidad del CBD y la idoneidad de su área para el desarrollo de la actividad comercial y de servicios lo hacen muy accesible, por la convergencia de las rutas de trasporte.

En la figura 5 se muestra el sistema de trasporte público urbano en Ciudad Obregón, articulado con 15 rutas, que sirven a la zona urba- 
na, así como a algunas localidades que la rodean. ${ }^{6}$ También aparecen algunas características de las rutas, como su dirección y el trayecto, a través de los principales centros de actividad. La mayor densidad de rutas de autobuses está en el centro de la ciudad, en donde se localiza el área comercial más importante (incluye Mercajeme) y mejor conectada, ya que las 15 rutas llegan a ella (véase figura 5).

\section{Figura 5}

Sistema de trasporte público urbano en Ciudad Obregón, 2014

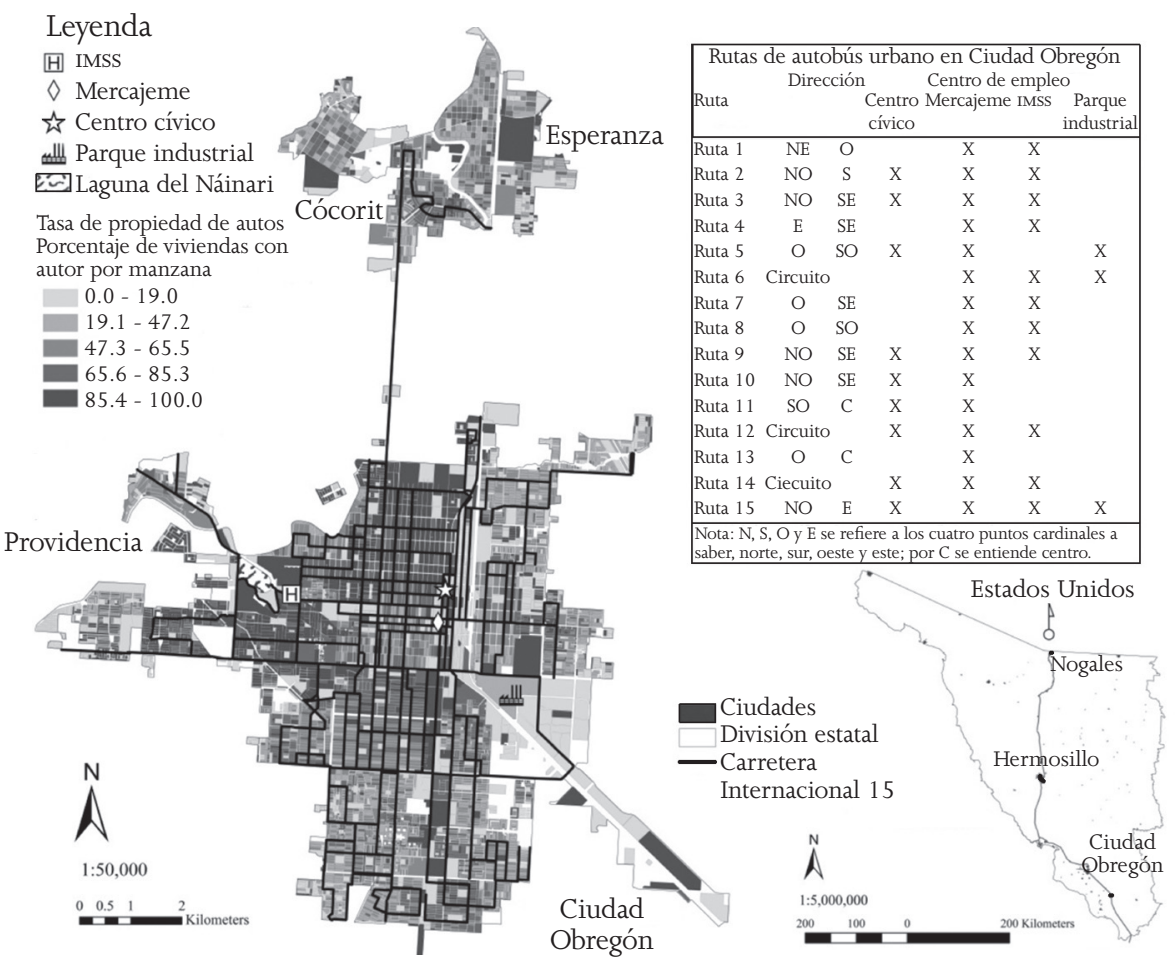

Fuente: elaborado con información del INEGI (2010b) y cartografía construida con base en trabajo de campo en la ciudad (de marzo a junio de 2014).

6 En la ciudad también confluyen rutas del sistema de trasporte suburbano y foráneo, que prestan servicio a los residentes en los trayectos en que ambos servicios se empalman. Sin embargo, para efectos de este estudio sólo se considera la provisión del trasporte urbano, pues la Ley 149 de Transporte para el Estado de Sonora prohíbe la prestación del servicio suburbano y foráneo en el área urbana (Congreso del Estado de Sonora 2013). 
En cambio, la zona industrial no está bien conectada con los lugares de residencia de los empleados y obreros; sólo tres líneas de autobuses pasan cerca de ella (véase figura 5). Los trabajadores deben tomar un segundo autobús, utilizar un vehículo particular o el de trasportación de la empresa para viajar al trabajo. La falta de cobertura del servicio en algunas áreas de la ciudad está vinculada con lo heterogéneo en la densidad de las rutas, ubicadas generalmente en la periferia, donde se localiza un mayor número de usuarios del trasporte público, ya que son pocos los que poseen auto, sobre todo los que viven al noreste y suroeste (véase figura 5).

La planeación, operación y regulación del sistema de trasporte público en Ciudad Obregón ha sido muy cuestionada en los últimos años. En Sonora el trasporte urbano, además de otras modalidades, se encuentra normado y regulado por la Ley 149 de Transporte para el Estado de Sonora, promulgada en 2002 (que derogó a la Ley 120 existente desde 1992), reformada en 2003, 2010, 2011 y 2013. Ésta supone la existencia de un sistema integrado por un programa estatal de trasporte y la coordinación entre la Secretaría de Infraestructura Urbana y Ecología y los municipios (Congreso del Estado de Sonora 2013). Además, éste debe contener los planes operativos de servicio (POS) por ciudad y las características de sustentabilidad, confort y calidad a las que habrá de apegarse la prestación del servicio público (artículo 18, Ley 149). Para la provisión de la trasportación urbana el servicio se concesiona a los particulares (artículo 57, Ley 149), mientras que las autoridades promueven e impulsan entre los concesionarios la integración de empresas y asociaciones de trasporte (artículo 37, Ley 149) (Congreso del Estado de Sonora 2013). En los últimos diez años se ha tratado de pasar de la provisión individual del servicio, con base en el esquema "hombre-camión", a una estrategia más integral con base en "hombre-ruta" o "ruta-empresa"7 En Ciudad Obregón ya se implementaron dos programas estatales, pero no han tenido el éxito esperado.

Por ejemplo, en 2006 se estableció el Programa de Modernización de Transporte del Gobierno del Estado (suBA), el cual logró asociar a

7 También se buscaba acabar con la "guerra del centavo", que se refiere a tener dos o más empresas en una misma ruta, para evitar que un chofer tratara de adelantar al otro para garantizar más pasajeros. 
los concesionarios del servicio de trasporte público en empresas integradoras, para aprovechar economías de escala e implementar tecnologías para el control del aforo y el recaudo. ${ }^{8}$ Aunado a ello, se buscó lo siguiente: a) el rediseño de rutas; b) la sustitución de unidades; c) el equipamiento e infraestructura; d) un sistema de pago y control de ingresos y e) una organización nueva (Landeros 2014; Ramos 2007).

Más tarde, con el cambio de gobierno se modificó la política de trasporte con la operación del programa Bus Sonora y la Norma de Calidad Bus Sonora, en 2011, se puso el acento en la prestación del servicio (calidad y atención al usuario). Entre los elementos novedosos, el Bus Sonora instaló un sistema de prepago, otorgó subsidios a estudiantes de hasta dos viajes gratis en semana laboral y en periodo escolar, además de otros criterios de calidad ligados a la infraestructura y a las unidades. El programa contemplaba ocho etapas de las cuales, de acuerdo con Landeros (2014), en diciembre de 2012 sólo dos se habían puesto en marcha: tarjeta de prepago y subsidio a estudiantes.

En un análisis reciente del marco institucional del servicio de trasporte público urbano en Sonora se señala que "la implementación de reglas y normas informales relacionadas a la rentabilidad del agente (concesionarios), las obligaciones de financiamiento adicionales del principal (Gobiernos del Estado) y la asimetría de información sobre los costos del servicio, provocó los conflictos en el transporte público urbano" (Landeros 2014, 149). A la fecha éstos continúan, y son agudizados por los paros del servicio en Hermosillo, Navojoa y Ciudad Obregón, donde opera el programa Bus Sonora.

En Sonora se vive una crisis del sistema de trasporte público en las principales ciudades, en donde éste falla cada vez que los trasportistas amenazan con una huelga, como consecuencia del incumplimiento del pago de los subsidios del gobierno del estado a los concesionarios o por la pugna de un aumento en las tarifas (Valenzuela 2013; Paredes y Navarro 2013). García (2006) y Landeros (2014) han documentado la problemática y los conflictos recurrentes. No obstante, los aspectos más sentidos por los usuarios en Ciudad Obregón son los

8 Referentes para ellos fueron el caso de Curitiba, Brasil, con la Red Integrada de Transporte, el padre de los sistemas de trasporte en América; desde su implementación, en 1974, ha sido un ejemplo para Colombia (Transmilenio), para Chile, con el Transantiago, el Sistema Integrado de Transporte de León, Guanajuato, y el Metrobús de la Ciudad de México. 
problemas asociados a la frecuencia con que pasan los autobuses y las condiciones de operación (Minjares 2012a y 2012b).

\section{Metodología}

Datos y construcción del sig para el trasporte público

Uno de los principales problemas con el trasporte público en países en vías de desarrollo es la falta de sistemas de información, sobre todo en las ciudades donde opera según el esquema "hombre-camión" o donde no ha sido posible instalar tecnología para contabilizar el aforo y conocer la demanda. Aunado a ello, la falta de estudios de movilidad con base en encuestas origen-destino, que sirvan para estimar la demanda potencial, es decir, la máxima, hace que el sistema de trasporte público sea un servicio poco eficiente (Casado 2008; Miralles-Guasch y Cebollada 2009). En México, los estudios de Fuentes (2009); Brugués y Rubio (2009); Salazar (2010); Casado (2014); Obregón-Biosca y Betanzo-Quezada (2015); Rosales (2012) y Rodríguez-Gámez y Tong (2012), entre otros, realizados con el esfuerzo conjunto de gobiernos, universidades y centros de investigación ofrecen un acercamiento al tema.

Debido a la connotación espacial en la composición de la demanda, los SIG constituyen una herramienta esencial para analizar la movilidad (Gutiérrez et al. 2008). Por ello, la metodología propuesta busca, con su ayuda y alimentada con información secundaria, estimar la demanda del servicio de trasporte público en Ciudad Obregón. El SIG se construyó a partir de la cartografía censal por manzana del INEGI (2010b), y del sistema de trasporte público generada en trabajo de campo, de marzo a junio de 2014. La información secundaria proviene también del INEGI (2010a), y comprende las características demográficas y socioeconómicas del censo reciente de población por manzana urbana.

Estimación de la demanda por manzana

Puesto que el trasporte público ofrece un servicio que se consume en el momento en que se produce, es muy importante estimar la de- 
manda con la mayor exactitud posible. Para ello, los métodos sugieren utilizar información de encuestas origen-destino o el aforo registrado en periodos anteriores para modelar la función de demanda ya conocida, y calcular elasticidades respecto al precio (Balcombe et al. 2004; Buehler y Pucher 2012; Ortúzar y Román 2003). Sin embargo, cuando esta información no está disponible, como en el caso de Ciudad Obregón, se sugiere un método más intuitivo para tener idea de las necesidades de movilidad y estimar la demanda potencial (Bazant 2011); para ello se diseñaron los pasos metodológicos siguientes:

1. Identificación de las necesidades de movilidad. Para identificar a la población con necesidad de movilizarse, se seleccionó a la mayor de 12 años por condición de actividad, ${ }^{9}$ y se clasificó en seis grupos: ocupados, desempleados abiertos, población dedicada a los quehaceres domésticos, estudiantes, pensionados y jubilados y otros. Se excluyó a los menores de 12 años puesto que quienes no asisten a la escuela tiene movilidad nula o dependen de las decisiones de un adulto; y aquéllos que sí van, además de depender de un adulto, acuden a planteles cercanos al lugar de residencia por lo que se desplazan a pie.

2. Selección del modo de trasporte. Se estableció el uso del automóvil privado y del trasporte público con base en probabilidades, según el número de viviendas particulares habitadas que disponen de auto o camioneta para cada manzana urbana. Además, se identificó la cantidad de viviendas en la manzana en donde los residentes tienen que usar el sistema de trasporte público como único modo de trasladarse, pues no cuentan con un automóvil privado. Al respecto, se asume que la tasa de motorización de la vivienda es de un ocupante por auto, ya que el INEGI no recoge información sobre el número de automóviles por vivienda.

9 Este segmento se divide en población económicamente activa y en la no económicamente activa. La primera comprende a los ocupados y desempleados abiertos; mientras que la segunda a las personas dedicadas a los quehaceres domésticos, estudiantes, pensionados y jubilados y otros. En esta última categoría se encuentran las que tienen alguna discapacidad y las no especificadas que requieren movilizarse en la ciudad (INEGI 2002). 
3. Prioridades de movilidad por grupo poblacional. Las perspectivas teóricas y los estudios sugieren que los trabajadores tienen mayor necesidad de movilizarse en la ciudad y, por tanto, requieren de un modo de trasporte que les ofrezca la posibilidad de hacerlo. Por ello, si el trabajador contara con un automóvil priorizaría su uso. Si existieran más viviendas con auto que trabajadores en una misma manzana, los autos restantes serían asignados al grupo de población inactiva de acuerdo con el siguiente orden de prioridad: amas de casa, estudiantes, jubilados, discapacitados y otros.

4. Estimación del número de viajeros (automovilistas y usuarios potenciales). Para cada manzana se calculó el total de viajeros por modo de trasporte y categoría de ocupación. La metodología permite, además, calcular el aforo y, al menos, comparar entre el auto y el trasporte público, como un medio para desplazarse por categoría de actividad (ocupados, amas de casa, estudiantes, jubilados, discapacitados, otros), y por sectores: manzanas, colonias y áreas geoestadísticas básicas.

5. Cálculo de la demanda potencial (viajes por semana laboral). Para el cálculo del aforo se establecieron las frecuencias de viaje por actividad de ocupación en la semana laboral (de lunes a viernes). Los trabajadores y estudiantes mayores de 12 años viajan los cinco días; pero se asume que las amas de casa tienen una frecuencia de viaje de tres días, mientras que el resto de los grupos se movilizan al menos un día en semana laboral. Asimismo, se estableció un viaje de ida y otro de vuelta a cada destino. Con estos nuevos criterios es posible establecer el aforo en función del número de viajes en una semana laboral, y después estimar el total de ellos por día.

\section{Operación del sistema de trasporte público Cobertura y accesibilidad}

Las 15 rutas con que cuenta la ciudad se encuentran distribuidas de tal manera que atienden a gran parte de la zona urbana. El sistema abarca 419 kilómetros lineales que, en una ciudad de 56.6 km², proporciona 
una cobertura de 7.4 kilómetros de red por cada $\mathrm{km}^{2}$ de área urbana poblada. La longitud promedio de cada ruta es de $27.9 \mathrm{~km}$, con una mínima de 17.3 (ruta 13, del centro hacia el oeste) y una máxima de 39.4 (ruta 9, que conecta al sureste con el Instituto Mexicano del Seguro Social, IMSS). La mayor parte de las rutas conectan las zonas norte y sur o este y oeste, y sólo algunas están diseñadas en circuito.

El SIG permite considerar un buffer de 250 metros de radio para cada ruta de autobús urbano, con el fin de analizar la cobertura en la provisión del servicio; hay un traslape de rutas en la mayoría de la red. La mayor densidad se aprecia en el centro cívico, el mercado municipal (Mercajeme) y el conglomerado de hospitales y clínicas del IMSS (véase parte izquierda de figura 6). Las rutas se han mantenido sin cambios sustanciales en los últimos años, pero los patrones de movilidad se modifican más a menudo, por ello es posible que la red sea ineficiente, y que se necesite hacer más trasbordos en los puntos con mayor afluencia, lo que dotaría de eficiencia y accesibilidad al sistema.

La accesibilidad del sistema de trasporte se define como la facilidad con la que las actividades en un mismo lugar se pueden alcanzar desde otra ruta (Liu y Zhu 2004). Con esta idea, en Ciudad Obregón se identifican diez zonas con desventaja de trasporte público ubicadas al noreste, suroeste y oeste de la periferia (véase parte izquierda de figura 6).También están las del interior de la ciudad, que permanecen inaccesibles o con baja densidad de la red. En las zonas no accesibles prevalece una tasa baja de propiedad de autos (véase figura 5) por lo que sus habitantes, por lo general de ingresos bajos, no tienen acceso a un auto y sufren las consecuencias de la mala planeación del trasporte (véase parte derecha de figura 6).

Los problemas de trasporte que enfrenta la población de bajos ingresos están relacionados con la inequidad en el acceso físico y económico al espacio (Vasconcellos 2010). Parte de las desventajas de vivir en ubicaciones periféricas se asocia con la lejanía de los lugares de trabajo, con el déficit de equipamiento público, así como de escuelas y centros de salud, y una oferta deficitaria de trasporte público. Si este último no está disponible en su barrio o no es eficiente, las familias deben comprar un auto para mejorar su movilidad y también su accesibilidad, situación a la que Gleeson y Randolph (2002) denominan "pobreza de trasporte". 


\section{Figura 6}

Cobertura del sistema de trasporte público urbano en Ciudad Obregón, 2014

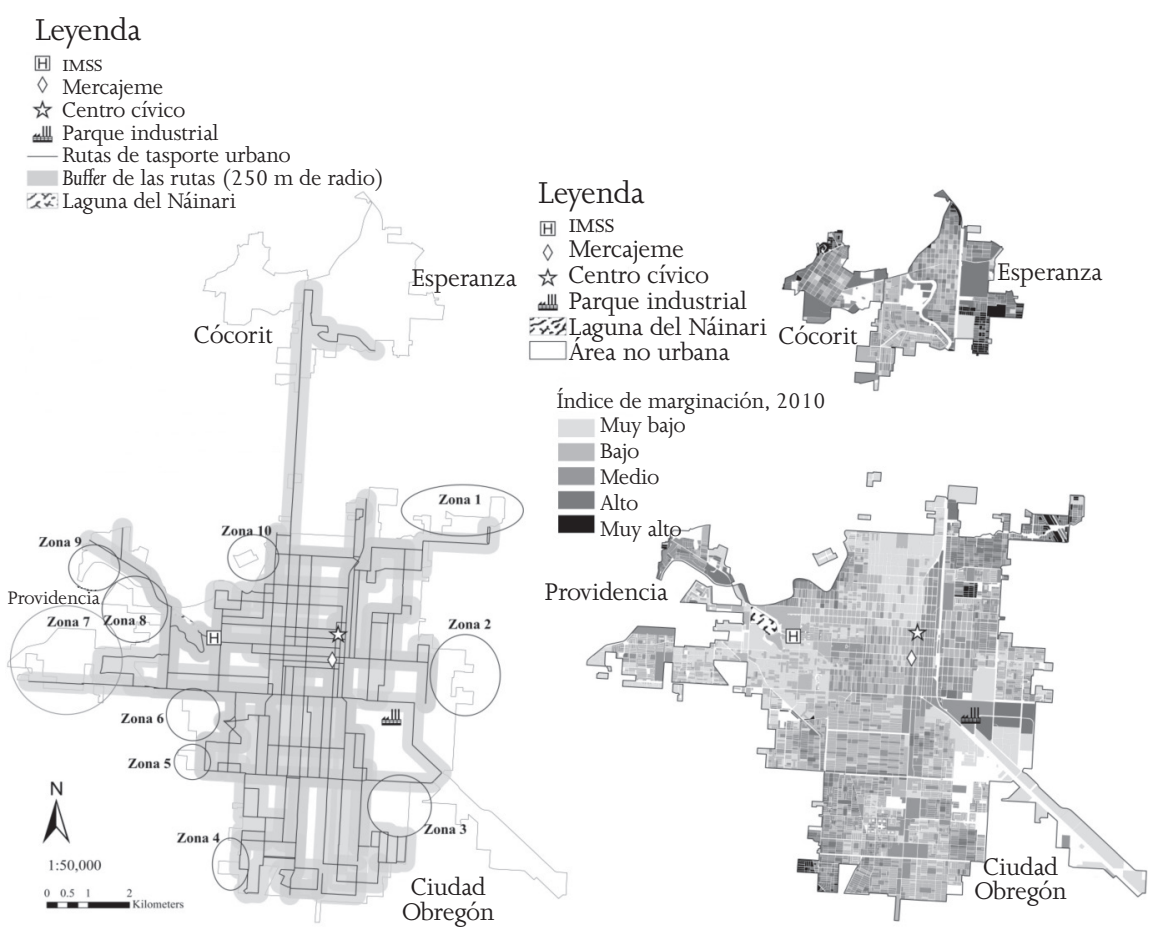

Fuente: elaborado con información del INEGI (2010b) y cartografía construida con base en trabajo de campo en la ciudad (de marzo a junio de 2014).

Estimación de la demanda del trasporte público y su provisión

Esta estructura en el sistema de rutas no refleja la demanda real, que es desconocida, ya que obliga a los usuarios a hacer trasbordos y pagar de nuevo la tarifa cada vez que se aborde una unidad. No obstante, permite tener una idea aproximada del volumen de viajes y, por tanto, de la demanda potencial. La metodología permite estimar el total de recorridos diarios en la semana laboral, con base en 2010, el cual ascendió a 201035 movimientos para el área conurbada de Ciudad 
Obregón-Providencia, y 231516 para ésta más su zona de influencia al norte, que incluye las localidades de Cócorit y Esperanza. La estimación puede ajustarse al crecimiento poblacional (registrado en el periodo 2010-2015), y proyectar la demanda.

El servicio de trasporte público tiene una demanda potencial de 73472 movimientos diarios en el área conurbada, y 86489 para la ciudad y su zona de influencia (véase figura 7). Al considerar la información de la encuesta Empleo y calidad de vida (COLSON 2009), que permite extraer datos precisos sobre los parámetros de movilidad de la población, definidos en la sección sobre la metodología, se encontró que la demanda de trasporte público es más o menos próxima a la estimación original con base en la información censal del INEGI (2010a): 75491 movimientos diarios para el área conurbada y 86 986 en la ciudad y su zona de influencia. Con estos parámetros, además es posible estimar los viajes en otros medios de trasporte motorizado (auto 62 508, pasajero en auto 23692 y 479 en motocicleta) y no motorizado (a pie 33464 y15 726 en bicicleta).

La oferta de trasporte público se percibe como uno de los elementos urbanos que permite incluir en la ciudad a la mayoría de los ciudadanos, a la vez que define la accesibilidad urbana. Por lo tanto, si el total de viajes en el área conurbada de Ciudad Obregón-Providencia fuera de 73 472, y la capacidad máxima de un autobús promedio en hora pico de 50 pasajeros (Banzant 2011, 180), se requerirían 1469 unidades para brindar el servicio; esta cifra hace referencia a las veces que la unidad deberá llenarse a su capacidad máxima para proveer el servicio.

Por otro lado, el Programa Operativo de Servicio (POS), que debe estar disponible para cada ciudad en apego a la Ley 149 (Congreso del Estado de Sonora 2013) debe detallar, entre otras cosas, los parámetros de servicio (por ejemplo la frecuencia con la que pasan los autobuses, el tamaño de la flota, los intervalos de hora pico y hora valle), que permitan estimar la oferta en apego al servicio deseado para la ciudadanía por la autoridad de trasporte. Sin embargo, el POS de Ciudad Obregón no se ha elaborado por las instancias correspondientes, por ello sólo es posible establecer escenarios para la provisión del servicio con base en las estimaciones de la demanda potencial. Por lo tanto, se calculó la flota necesaria para proveer el servicio cuando 
la frecuencia es de 10 minutos en hora pico y de 20 en hora valle, ${ }^{10}$ y la duración promedio de la vuelta es 47 minutos. ${ }^{11}$ En función de los horarios de despacho, que considera 15 minutos entre una vuelta y otra, cada lado de la ruta tiene que ofrecer 73 viajes. Esto implica que se requieren siete unidades por lado, de cada ruta, lo que incluye una emergente. Así, el sistema requiere 210 autobuses para proveer el servicio en Ciudad Obregón.

Figura 7

Demanda potencial por trasporte privado y público en Ciudad Obregón, 2010

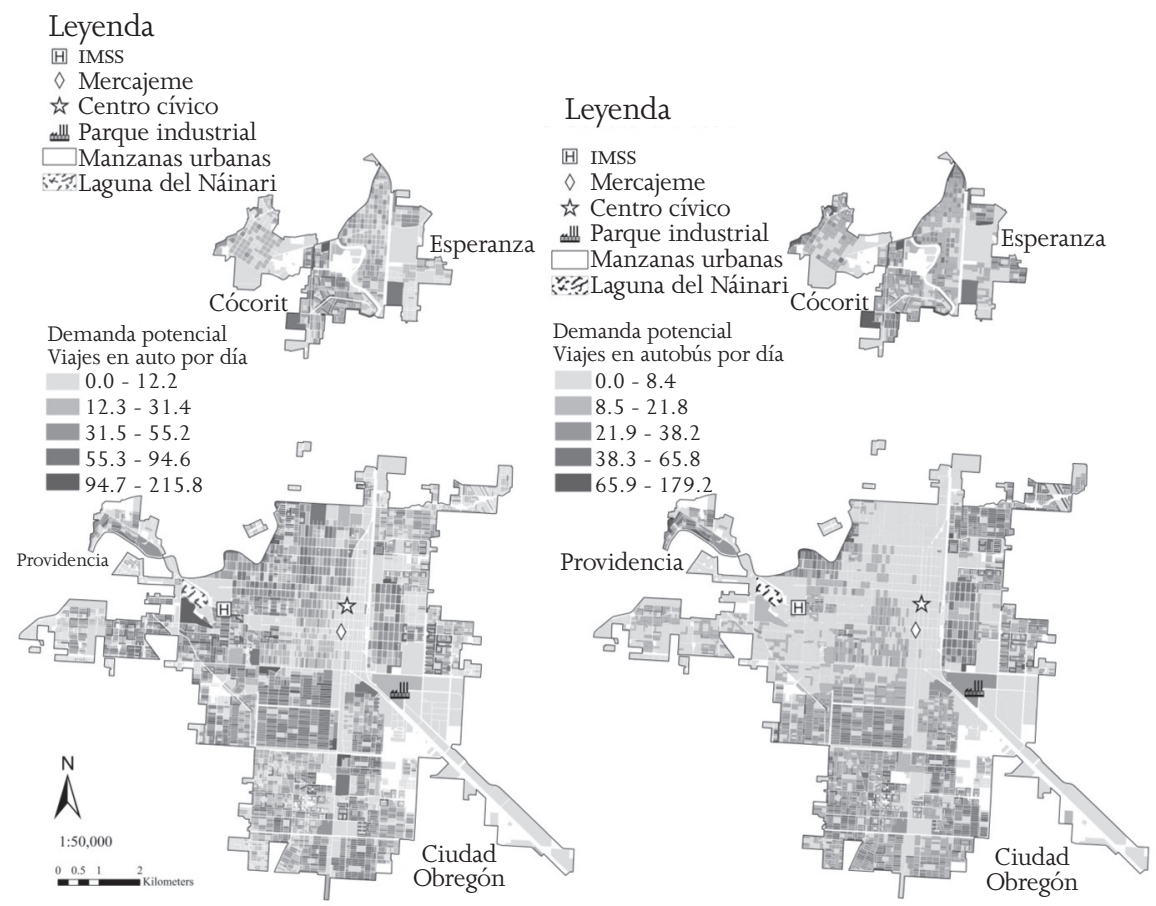

Fuente: elaborado con información del INEGI (2010b) y con base en la metodología descrita.

10 El horario de servicio considerado fue de 5:30 a 22:30 (última salida), y para la hora pico de $5: 30$ a 9:00; $12: 30$ a $15: 30$ y de $18: 00$ a 20:30.

11 Si bien la longitud promedio de una ruta es de $28 \mathrm{~km}$ (ida y vuelta), debe considerarse que cada una tiene dos lados, y que el servicio se empieza a ofrecer en ambos extremos al mismo tiempo, a una velocidad promedio de $18 \mathrm{~km}$ por hora. 
Tras la desintegración, en 2012, de la empresa Sistema Integrado de Transporte Obregón-Concentradora de Cajeme del Transporte Público entraron en operación cuatro: a) Transporte Moderno de Obregón; b) Transportes Urbanos y Especiales de Cajeme; c) Transportes Integrados de Cajeme y d) Integradora de Transporte del Sur de Sonora (Minjares 2012c). El número de unidades que oferta el servicio ha sido muy inestable, en 2012 operaban 154 autobuses (Valdivia 2012; Minjares 2012b), lo que da cuenta del déficit en la provisión del servicio, que requiere ser subsanado.

\section{Consideraciones finales}

La metodología empleó dos elementos sustanciales para la estimación de la demanda potencial del trasporte público: a) la estructura familiar y las actividades cotidianas que llevan a cabo sus miembros, elementos que inciden en la organización de los desplazamientos y en los medios de trasporte que utilizan y b) la accesibilidad a los modos de trasporte, a partir de que no toda la población tiene la misma prioridad de acceso. Así, ante la falta de una encuesta origen-destino y un estudio de movilidad, la estimación de la demanda potencial es un primer ejercicio exploratorio.

En Ciudad Obregón se desconoce la demanda de trasporte público, puesto que los programas de modernización no han logrado implementar y mantener una flota de unidades con la tecnología necesaria para el registro del aforo. Por ello, la metodología propuesta logró estimar la demanda potencial de usuarios que, al compararla con la oferta esperada, se observó un desequilibrio claro en la provisión del servicio, lo que refleja la grave situación del trasporte urbano, pues incluso la ciudad presenta una tasa menor de motorización de vehículos privados, así como de propiedad de autos por vivienda.

Quedan pendientes algunos temas, por ejemplo la construcción de escenarios para modelar la oferta de trasporte público y establecer el equilibrio o desequilibrio en la provisión del servicio considerando las diferencias en la demanda por ruta. $\mathrm{Al}$ respecto, el programa estatal de trasporte y el pos de Ciudad Oregón son documentos indispensables para planear el sistema de trasporte público, que las autoridades 
correspondientes deben elaborar de inmediato. Por ello, la estimación realizada aquí es una base para su planeación, sobre la cual deben hacerse cálculos y proyecciones nuevas tomando en cuenta dos situaciones: el aumento de la población y los cambios rápidos en las características demográficas y socioeconómicas, que modifican la naturaleza y características de los desplazamientos al trabajo y aumentan/disminuyen la demanda de viajes continuamente, para lo cual el sistema de trasporte debe estar preparado.

Recibido en marzo de 2015 Aceptado en mayo de 2015

\section{Bibliografía}

Balcombe, Richard, Roger Mackett, Neil Paulley, John Preston, Jeremy Shires, Helena Titheridge, Mark Wardman y Peter White. 2004. The demand for public transport: a practical guide. TRL Report, TRL593. http://www.demandforpublictransport.co.uk/TRL593. pdf (15 de noviembre de 2014).

Baum, Charles. 2009. The effects of vehicle ownership on employment. Journal of Urban Economics LXVI (3): 151-163.

Bazant, Jan. 2011. Planeación urbana estratégica. Mexico: Trillas.

Brugués, Alejandro y Rodolfo Rubio. 2009. Movilidad intra-urbana en ciudades medias del estado de Sinaloa. Ponencia presentada en el primer Congreso de egresados de El Colegio de la Frontera Norte, Tijuana. http://www.academia.edu/1455581/ Movilidad_intra-urbana_en_ciudades_medias_del_estado_de_ Sinaloa_?login=\&email_was_taken=true\&login=\&email_was_ taken=true (3 de marzo de 2014).

Buchanan, Nick, Ross Barnett, Simon Kingham y Doug Johnston. 2006. The effect of urban growth on commuting patterns in Christchurch, New Zealand. Journal of Transport Geography XVI (5): 342-354. 
Buehler, Ralph y John Pucher. 2012. Demand for public transport in Germany and the USA: an analysis of rider characteristics. Transport Reviews XXXII (5): 541-567.

CAF. 2011. Desarrollo urbano y movilidad en América Latina. Banco de Desarrollo de América Latina. http://omu.caf.com/media/30839/desarrollourbano_y_movilidad_americalatina.pdf (12 de marzo de 2014).

CAF. 2009. Observatorio de Movilidad Urbana para América Latina. Información para mejores políticas y mejores ciudades. Banco de Desarrollo de América Latina. http://publicaciones.caf.com/media/1150/2.pdf (3 de marzo de 2014).

Casado Izquierdo, José María. 2014. Patrones horarios de la movilidad cotidiana en la zona metropolitana del valle de México, 19942007. Scripta Nova XVIII (487). http://www.ub.edu/geocrit/sn/sn487.htm (13 de agosto de 2014).

Casado Izquierdo, José María. 2008. Estudios sobre movilidad cotidiana en México. Scripta Nova XII (273). http:/ /www.ub.es/geocrit/ sn/sn-273.htm (22 de agosto de 2014).

Cebollada i Frontera, Ángel. 2009. Mobility and labour market exclusion in the Barcelona metropolitan region. Journal of Transport Geography XVII (3): 226-233.

COLSON. 2009. Encuesta empleo y calidad de vida. Módulos de residentes, vivienda y empleo. Archivos electrónicos.

Congreso del Estado de Sonora. 2013. Ley 149 de Transporte para el Estado de Sonora. Hermosillo, Sonora. http://www.congresoson. gob.mx/Leyes_Archivos/doc_59.pdf (23 de abril de 2014).

Figueroa, Oscar. 2005. Transporte urbano y globalización. Políticas y efectos en América Latina. Eure Xxxi (94): 41-53. 
Flechas, Ana Luisa. 2006. Movilidad y transporte: un enfoque territorial. Facultad de Ingeniería. Universidad Nacional de Colombia. Bogotá.

Fuentes, César Mario. 2009. La estructura espacial urbana y accesibilidad diferenciada a los centros de empleo en Ciudad Juárez, Chihuahua. región y sociedad XXI (44): 117-144.

García Román, Omar. 2006. El Servicio Público de Transporte Urbano en la ciudad de Hermosillo. Análisis de los procedimientos administrativos de la concesión 1982-2004. Tesis de maestría en ciencias sociales, COLSON.

Gautier, Pieter eYves Zenou. 2010. Car ownership and the labor market of ethnic minorities. Journal of Urban Economics LXVII (3): 392-403.

Gleeson, Brendan y Bill Randolph. 2002. Social disadvantage and planning in the Sydney context. Urban Policy and Research Xx (1): 101-107.

Gordon, Peter y Harry Richardson. 1996. Beyond polycentricity: the dispersed metropolis, Los Angeles, 1970-1990. Journal of the American Planning Association LXII (3): 289-295.

Gutiérrez, Javier, Osvaldo Cardozo y Juan Carlos García. 2008. Modelos de demanda potencial de viajeros en redes de transporte público: aplicaciones en el metro de Madrid. Proyección 4 (1). http://bdigital.uncu.edu.ar/objetos_digitales/3242/gutierrezproyeccion4. pdf (7 de mayo de 2015).

Illes, Richard. 2005. Public transport in developing countries. Países Bajos: Elsevier.

INEGI. 2014. Consulta interactiva del Sistema Estatal y Municipal de Bases de Datos (SIMBAD). http://sc.inegi.org.mx/cobdem/consulta-por-ageo.jsp?recargar=true (26 de noviembre de 2014). 
INEGI. 2010a. Censo de población y vivienda 2010. http://www3. inegi.org. $\mathrm{mx} /$ sistemas $/$ temas $/$ default.aspx? $=$ est\&c $=17484>$ (25 de septiembre de 2014).

INEGI. 2010b. Cartografía urbana de Ciudad Obregón. Censo de población 2010. Disco compacto. Aguascalientes, México. INEGI.

INEGI. 2009. Encuesta nacional sobre uso del tiempo (ENUT). Aguascalientes, México. http://www.inegi.org.mx/est/contenidos/Proyectos/Encuestas/Hogares/especiales/enut/enut2009/default. aspx (25 de enero de 2015).

INEGI. 2002. Guía de conceptos, uso e interpretación de la estadística sobre la fuerza laboral en México. http://www.inegi.org.mx/est/ contenidos/espanol/metodologias/otras/guia.pdf (17 de junio de 2014).

Jaramillo, Ciro, Carmen Lizárraga y Alejandro Luis Grindlay. 2012. Spatial disparity in transport social need and public transport provision in Santiago de Cali (Colombia). Journal of Transport Geography XXIV (1): 340-357.

Landeros Jaime, Francisco Javier. 2014. El marco institucional del servicio de trasporte público urbano en Hermosillo, Sonora: análisis del subsidio y el conflicto entre concesionarios y el gobierno del estado 2009-2012. Tesis de maestría en ciencias sociales, COLSON.

Lazo Corvalán, Alejandra. 2008. Transporte, movilidad y exclusión. El caso de Transantiago en Chile. Scripta Nova (Revista Electrónica de Geografía y Ciencias Sociales) XII (270). http://www.ub.edu/ geocrit/sn/sn-270/sn-270-45.htm (13 de mayo de 2014).

Liu, Suxia y Xuan Zhu. 2004. Accessibility analyst: an integrated GIS tool for accessibility analysis in urban transportation planning. Environment and Planning B: Planning and Design XXXI (1): 105-124.

Minjares, Francisco. 2012a. Crece conflicto en sector transporte. Tribuna delYaqui.http://www.tribuna.info/index.php?option=com_conten 
t\&view=article\&id=221434:n1p1\&cati $\mathrm{d}=4$ :ciudad\&Itemid $=131$ (15 de octubre de 2012).

Minjares, Francisco. 2012b. El transporte afecta a usuarios: pésimo servicio. Tribuna del Yaqui. http://www.tribuna.info/index.php? option=com_content\&view=article\&id=264889:n1p1\&cati d=4: ciudad\&Itemid=131 (10 de diciembre de 2012).

Minjares, Francisco. 2012c. Urgen se mejore el transporte urbano. Tribuna del Yaqui. http://www.cdobregon.gob.mx/opinion/sintesis/20121112/urgen_se_mejore_el_transporte_urbano/urgen_ se_mejore_el_transporte_urbano.htm (9 de mayo de 2012).

Miralles-Guasch, Carme y Ángel Cebollada i Frontera. 2009. Movilidad cotidiana y sostenibilidad. Una interpretación desde la geografía humana. Boletín de la A.G.E. 50: 193-216.

Miralles-Guasch, Carme y Ángel Cebollada i Frontera. 2003. Movilidad y transporte: opciones políticas para la ciudad. Fundación Alternativas. http://www.falternativas.org/content/download/5686 /163153/file/fa70_15-11-06_doc25.pdf (19 de noviembre de 2014).

Montezuma, Ricardo. 2003. Ciudad y transporte: la movilidad urbana. En La ciudad inclusiva. Cuaderno de la CEPAL no. 88, compilado por Marcello Balbo, Ricardo Jordán y Daniela Simioni, 175-192. Santiago de Chile: Naciones Unidas.

Negrete, María Eugenia y Catherine Paquette. 2011. La interacción entre transporte público y urbanización en la zona metropolitana de la Ciudad de México: un modelo expansivo que llega a sus límites. Territorios XXV: 15-33.

Obregón-Biosca, Saúl Antonio y Eduardo Betanzo-Quezada. 2015. Análisis de la movilidad urbana de una ciudad media mexicana, caso de estudio: Santiago de Querétaro. Economía, Sociedad y Territorio XV (47): 61-98. 
Ortúzar, Juan de Dios y Luis Willumsen. 2011. Modeling tranport. Reino Unido: John Wiley and Sons, Ltd.

Ortúzar, Juan de Dios y Concepción Román. 2003. El problema de modelación de demanda desde una perspectiva desagregada: el caso del transporte. Eure XXIX (88): 149-171.

Paredes, Luz del Carmen y Francisco Navarro. 2013. Caos por paralización de transporte urbano en Cajeme. Diario del Yaqui. http:// diariodelyaqui.mx/n/index.php?option=com_content\&view $=\mathrm{ar}$ ticle\&id=25213: caos-por-paralizacion-de-transporte-urbano-en cajeme\&catid=13:principal\&Itemid=127 (24 de enero de 2013).

Pucher, John. 2004. Public transportation. En The geography of urban transportation, coordinado por Susan Hanson y Genevieve Giuliano, 199236. Nueva York: The Guilford Press.

Ramos, José Antonio. 2007. Programa de Modernización del Sistema de Transporte Público del Estado de Sonora “subA”. Transporte Urbano Público. http://transporteurbanopublico.wordpress. com/2007/08/30/programa-de-modernizacion-del-sistemade-transporte-publico-del-estado-de-sonora-suba/ (6 de abril de 2014).

Rodríguez-Gámez, Liz Ileana y Daoqin Tong. 2012. New perspectives on the spatial analysis of urban employment distribution and commuting patterns: the cases of Hermosillo and Ciudad Obregon, Mexico. Tesis de doctorado en geografía, Universidad de Arizona.

Rosales, Raúl. 2012. Desplazamientos por motivo de trabajo en Nogales, Sonora. Patrones de comportamiento, 2008. Tesis de maestría en ciencias sociales, COLSON.

Rouwendal, Jan y Peter Nijkamp. 2004. Living in two worlds: a review of home-to-work decisions. Growth and Change XXXv (3): 287-303.

Salazar, Clara. 2010. Dos sistemas de movilidad cotidiana en la Ciudad de México: domicilio-escuela y domicilio-trabajo. En Los grandes 
problemas de México. Desarrollo urbano y regional, coordinado por Gustavo Garza y Martha Schteingart, 511-550. México: El Colegio de México.

Schwanen, Trim, Frans M. Dieleman y Martin Dijst. 2004. The impact of metropolitan structure on commute behavior in The Netherlands: a multilevel approach. Growth and Change Xxxv (3): 304-333.

Secretaría de Gobierno del Estado de Sonora. 2012. Consejo Ciudadano para la Sustentabilidad del Transporte. Hermosillo, Sonora. Documento interno en Power Point (creado el 26 de noviembre de 2012).

Secretaría de Tránsito y Transporte de Bogotá. 2012. Transporte Público-V8. Formulación del Plan Maestro de Movilidad para Bogotá D. C. Alcaldía Mayor de Bogotá D.C. y Duarte Guterman \& Cia. Ltda. http://www.movilidadbogota.gov.co/hiwebx_archivos/ ideofolio/08-TransportePublico_15_9_24.pdf (15 de noviembre de 2014).

Valdivia, Mónica. 2012. Llega nueva empresa de transporte público. InfoCajeme. http://www.infocajeme.com/noticias.php?id=18963 (20 de junio de 2012).

Valenzuela, Gabriela. 2013. Siguen los problemas de transporte público en Obregón. Azteca Sonora. http://www.aztecasonora.com/ noticias/sonora-sur/general/14431-siguen-los-problemas-detransporte-p\%C3\%BAblico-en-obreg\%C3\%B3n.html (24 de enero de 2013).

Vasconcellos, Eduardo. 2010. Análisis de la movilidad urbana. Espacio, medio ambiente y equidad. Bogotá: Corporación Andina de Fomento.

Vega, Amaya y Aisling Reynolds-Feighah. 2008. Employment sub-centers and travel-to-work mode choice in the Dublin region. Urban Studies XLV (9): 1747-1768. 
